Vol. 47 (1993) [385-393]

\title{
GAP TAUBERIAN THEOREMS
}

\author{
JEFF CONNOR
}

\begin{abstract}
In the first section we establish a connection between gap Tauberian conditions and isomorphic copies of $c_{0}$ for perfect coregular conservative BK spaces and in the second we give a characterisation of gap Tauberian conditions for strong summability with respect to a nonnnegative regular summability matrix. These results are used to show that a gap Tauberian condition for strong weighted mean summability is also a gap Tauberian condition for ordinary weighted mean summability. We also make a remark regarding the support set of a matrix and give a Tauberian theorem for a class of conull spaces.
\end{abstract}

In this note we make some remarks regarding gap Tauberian theorems in the context of some settings associated with strong and ordinary summability. In the first part of this paper we provide an interpretation that leads to the construction of isomorphic copies of $c_{0}$ and establish a partial converse. In the second we characterise the gap Tauberian conditions for strong summability with respect to a nonnegative regular summability matrix. In a later section we restate this characterisation in terms of the support set of the matrix. We also apply these results to weighted mean summability methods and, as a bonus, we establish an analog of a gap Tauberian theorem for a large collection of conull spaces.

We use the standard notation of summability and Banach space theory as it is found in [4] and [13]. Let $\omega=$ all scalar sequences $\}, \varphi=\{x \in \omega: x$ is finitely nonzero $\}$ and let $c_{0}, c$, and $l_{\infty}$ denote the vector subspaces of $\omega$ consisting of, respectively, the null sequences, the convergent sequences and the bounded sequences. We also let $e^{k}=$ the $k$ th unit vector, $e=(1,1,1, \ldots)$, and, if $E$ is a Banach space, we let $E^{\prime}$ denote its continuous dual. If $S$ is a subset of a Banach space $E$, we let $[S]_{E}$ denote the closed linear span of $S$ in $E$. A $B K$ space is a Banach space which is a vector subspace of $\omega$ that has the property the inclusion map from $E$ into $\omega$ is continuous when $\omega$ is given the topology of coordinatewise convergence. A BK space $E$ is said to be conservative if $c \subset E$ and is said to be perfect if $[c]_{E}=E$. We recall that if $E$ and $F$ are BK spaces and $E$ is contained in $F$, then the inclusion map from $E$ into $F$ is continuous.

Received 7th May, 1992.

Research supported by an Ohio University Research Council Grant.

Copyright Clearance Centre, Inc. Serial-fee code: 0004-9729/93 \$A2.00+0.00. 
An infinite array of scalars $T=\left(t_{n, k}\right)_{n, k=1, \infty}$ is called a regular summability matrix provided $\lim _{n} \sum_{k} t_{n, k} x_{k}=\lim _{k} x_{k}$ whenever $x=\left\langle x_{k}\right\rangle \in c$. The matrix $T$ is called a triangle if $t_{n, n} \neq 0$ for all $n \in N$ and $n<k$ implies $t_{n, k}=0$. If $x \in \omega$ and $\sum_{k} t_{n, k} x_{k}$ exists for all $n \in \mathrm{N}$, we let $T x$ denote the sequence $\left\langle\sum_{k} t_{n, k} x_{k}: n \in \mathrm{N}\right\rangle$. Let $c_{T}=\{x \in \omega: T x \in c\} . c_{T}$ is called the convergence domain of $T$ and if $x \in c_{T}$ we say that $x$ is $T$-summable and set $T-\lim x=\lim _{n} \sum_{k} t_{n, k} x_{k}$. It is well-known that if $T$ is a triangle, then $c_{T}$ is a BK space with the norm $\|x\|_{T}=\sup _{n}\left|\sum_{k} t_{n, k} x_{k}\right|=\|T x\|_{\infty}$.

Throughout this note we let $\gamma: \mathbf{N} \cup\{0\} \rightarrow \mathbf{N} \cup\{0\}$ denote an increasing function with $\gamma(0)=0$ and set

$$
G(\gamma)=\left\{x \in \omega: x_{k}-x_{k+1} \neq 0 \text { implies there exists } r \in \mathbb{N} \text { such that } k=\gamma(r)\right\} .
$$

A gap Tauberian theorem for a matrix summability method $T$ generally asserts that if $\gamma$ satisfies some condition depending on $T, x \in G(\gamma)$ and $T x \in c$ then $x$ is convergent. With this in mind, we say that, for a sequence space $E, G(\gamma)$ is a gap Tauberian condition for $E$ if $E \cap G(\gamma) \subset c$. For a given $\gamma$, we set $b^{r}(\gamma)=\sum_{i>\gamma(r)}^{\gamma(r+1)} e^{i}$.

\section{Gap TAUBERIAN CONDITIONS AND ISOMORPHIC COPIES OF $c_{0}$}

The results in this section follow from the fundamental properties of BK spaces and the Bessaga-Pelcznyski characterisation of Banach spaces containing isomorphic copies of $c_{0}$.

THEOREM 1.1. Let $E$ be a conservative $B K$ space and $\gamma$ an increasing sequence of integers with $\gamma(0)=0$. If $G(\gamma)$ is a gap Tauberian condition for $E$ and $\left\langle b^{r}(\gamma)\right\rangle$ is basic in $E$, then $\left\langle b^{r}(\gamma)\right\rangle$ is equivalent to $c_{0}$ 's unit vector basis and the norm of $E$ and $c_{0}$ are equivalent on $Z=\left[\left\langle b^{r}(\gamma)\right\rangle\right]_{E} \subset c_{0}$.

Proof: Let $b^{r}=b^{r}(\gamma)$. First note that $\inf _{r}\left\|b^{r}\right\|_{E}>0$. If not, then there is a subsequence $\left\langle b^{r_{l}}\right\rangle$ of $\left\langle b^{r}\right\rangle$ such that $\left\|b^{r_{l}}\right\|_{E}<2^{-i}$ for each $l \in \mathbf{N}$. Now $x=\sum_{l} b^{r_{l}} \in E$ and it can be arranged for $x$ to have both 0 and 1 as limit points. As $x \in G(\gamma) \cap E$ and $x \notin c, G(\gamma)$ is not a gap Tauberian condition for $E$.

Observe that, since $c_{0} \subset E$ and the inclusion map is continuous, $\sum_{r}\left|f\left(b^{r}\right)\right|<\infty$ for all $f \in E^{\prime}$. Since $\left\langle b^{r}\right\rangle$ is basic in $E,\left\langle b^{r}\right\rangle$ is equivalent to $c_{0}$ 's unit vector basis [4, p.45].

The remainder of the claim follows from noting that $Z \subset c_{0}$ and that the inclusion maps from $Z$ into $c_{0}$ and from $c_{0}$ into $E$ are each continuous. 
The converse of the preceding proposition is obviously false: If $E=l_{\infty}$ and $\gamma(r)=r$ then $\left\langle b^{r}(\gamma)\right\rangle$ is basic, $Z=c_{0}$ and $G(\gamma) \cap l_{\infty}=l_{\infty}$. We can, however, establish a partial converse by making suitable restrictions on $E$. First, in order to avoid our counterexample, we assume that $E$ is perfect. Also recall that if $G(\gamma)$ is a gap Tauberian condition for a conservative BK space $E$, then $E$ must be coregular, that is, there must be an $f \in E^{\prime}$ such that $f(e) \neq \sum_{k} f\left(e^{k}\right)[3]$.

A BK space $E$ is said to have a monotone norm if, for all $x=\left\langle x_{k}\right\rangle \in E$ and $n \in \mathrm{N}$, $\left\|\sum_{k=1}^{n} x_{k} e^{k}\right\|_{E} \leqslant\left\|\sum_{k=1}^{n+1} x_{k} e^{k}\right\|_{E}$ and $\|x\|_{E}=\sup _{n}\left\|\sum_{k=1}^{n} x_{k} e^{k}\right\|_{E}$. Observe that if $E$ has a monotone norm, then $\left\langle b^{r}(\gamma)\right\rangle$ is basic for any $\gamma[4, \mathrm{p} .36]$ and $[\varphi \cap G(\gamma)]_{E}=[\varphi]_{E} \cap G(\gamma)$.

TheOREM 1.2. Let $E$ be a perfect coregular BK space with a monotone norm. Then $G(\gamma)$ is a gap Tauberian condition for $E$ if and only if $\left\langle b^{r}(\gamma)\right\rangle$ is equivalent to $c_{0}$ 's unit vector basis.

Proof: Since $E$ has a monotone norm, we have that $\left\langle b^{r}(\gamma)\right\rangle$ is basic in $E$ and hence, if $G(\gamma)$ is a gap Tauberian condition for $E$, the preceding result yields that $\left\langle b^{r}(\gamma)\right\rangle$ is equivalent to $c_{0}$ 's unit vector basis.

Now suppose that $\left\langle b^{r}(\gamma)\right\rangle$ is equivalent to $c_{0}$ 's unit vector basis. Note that if $w \in G(\gamma) \cap\left[\left\langle e^{k}\right\rangle\right]_{E}=\left[\left\langle b^{r}(\gamma)\right\rangle\right]_{E}$, then there is a $z=\left\langle z_{r}\right\rangle \in c_{0}$ such that $w=\sum_{r} z_{r} b^{r}$ and hence $w \in c_{0}$.

Next note that, since $E$ is coregular and $\left\langle e^{k}\right\rangle$ is basic in $E, e \notin[\varphi]_{E}$. Since $E$ is perfect, it follows that if $x \in E$, then there is a scalar $\lambda_{x}$ and a $w_{x} \in[\varphi]_{E}$ such that $x=\lambda_{x} e+w_{x}$. If $x \in G(\gamma) \cap E$, then $w_{x} \in G(\gamma) \cap[\varphi]_{E}$ and hence $x$ is convergent to $\lambda_{\mathbf{x}}$.

Corollary 1.3. Every perfect coregular conservative BK space with a monotone norm has a gap Tauberian condition.

Proof: Observe that, since $E$ is coregular, $\sum_{k} e^{k}$ does not converge in $E$. It follows that there is a $\gamma$ and a $\delta>0$ such that

$$
\left\|\sum_{k>\gamma(r)}^{r(r+1)} e^{k}\right\|>\delta
$$

for all $r \in N$. Now, since $E$ is conservative, $\sum_{r}\left|f\left(b^{r}(\gamma)\right)\right|<\infty$ for all $f \in E^{\prime}$. This is enough to insure that $\left\langle b^{r}(\gamma)\right\rangle$ is equivalent to $c_{0}$ 's unit vector basis and hence $G(\gamma)$ is a gap Tauberian condition for $E$.

Corollary 1.4. Let $T$ be a triangular regular matrix summability matrix such that $c_{T}$ is a $B K$ space with a monotone norm. Then there is a subspace $W$ of $c_{0}$ such that $T$ restricted to $W$ is an isomorphism onto its range. 
Proof: Since $T$ is a regular triangle, Fridy [6] has shown that there is a $\gamma$ such that $G(\gamma)$ is a gap Tauberian condition for $c_{T}$. Now, since $\left\langle b^{r}(\gamma)\right\rangle$ is equivalent to $c_{0}$ 's unit vector basis in $c_{T},\left\langle T b^{r}(\gamma)\right\rangle$ is equivalent to $c_{0}$ 's unit vector basis in $c_{0}$. Let $W=\left[\left\langle b^{r}(\gamma)\right\rangle\right]_{c_{0}}$. Since $\left\langle b^{r}(\gamma)\right\rangle$ and $\left\langle T b^{r}(\gamma)\right\rangle$ are both equivalent to $c_{0}$ 's unit vector basis, $T$ acts as an isomorphism from $W$ onto $T(W)$.

\section{GaP TAUBERIAN THEOREMS FOR STRONG SUMMABILITY}

In this section we characterise gap Tauberian conditions for strong summability with respect to nonnegative regular summability matrices. Before giving our characterisation, we recall a few definitions and facts.

If $T$ is a nonnegative regular summability method, $x \in \omega$ and $\lambda$ is a scalar, we say that $x$ is strongly $T$-summable to $\lambda$ if $\lim _{n} \sum_{k} t_{n, k}\left|x_{k}-\lambda\right|=0$. If $A \subset \mathrm{N}$, we let $I_{A}$ denote the characteristic function of $A$. Finally we recall the following result [10, $4.1,5]$.

ThEOREM 2.1. Let $T$ be a nonnegative regular summability method. If $x$ is strongly $T$-summable to $\lambda$, then there is a subset $A$ of $\mathrm{N}$ such that $T-\lim I_{A}=1$ and $\lim _{k}\left(x_{k}-\lambda\right) I_{A}(k)=0$.

Theorem 2.2. Let $T$ be a nonnegative regular summability method. Then the following statements are equivalent.

(1) $G(\gamma)$ is a gap Tauberian condition for strong $T$-summability.

(2) For all $A \subset \mathrm{N}$ such that $T-\lim I_{A}=1,(\gamma(r), \gamma(r+1)] \cap A \neq \emptyset$ for all but finitely many $r \in \mathbb{N}$.

(3) $\quad \lim \sup \sum_{r} \sum_{k>\gamma\left(n_{r}\right)}^{\gamma\left(n_{r}+1\right)} t_{n, k}>0$ for all increasing subsequences $\left\langle n_{r}\right\rangle$ of natural numbers.

Proof: (1) implies (2): Suppose that (2) fails and that we have found $A \subset \mathbf{N}$ such that $T-\lim I_{A}=1$ and an increasing sequence $\left\langle n_{r}\right\rangle$ of natural numbers such that $A \cap\left(\gamma\left(n_{r}\right), \gamma\left(n_{r}+1\right)\right]=\emptyset$ for all $r \in N$. Set $B=\bigcup_{r \in N}\left(\gamma\left(n_{r}\right), \gamma\left(n_{r}+1\right)\right]$ and let $x=I_{B}$. Since $B \subset N-A, x$ is strongly $T$-summable to 0 and has both 0 and 1 as limit points. Since $x \in G(\gamma), G(\gamma)$ is not a gap-Tauberian condition for strong $T$-summability.

(2) implies (1): Now suppose that $\gamma$ satisfies (2), $x$ is strongly $T$-summable and $x \in G(\gamma)$. Without loss of generality, suppose that $x$ is strongly $T$-summable to 0 . Select $A \subset \mathrm{N}$ such that $T-\lim I_{A}=1$ and $\lim x_{k} I_{A}(k)=0$.

Let $\beta: \mathbf{N} \rightarrow \mathbf{N}$ be an arbitrary increasing function. Select an increasing sequence of integers $\left\langle n_{l}\right\rangle$ such that $\left(\gamma\left(n_{l}\right), \gamma\left(n_{l}+1\right)\right] \cap\{\beta(r): r \in N\} \neq 0$ and 
$\left(\gamma\left(n_{l}\right), \gamma\left(n_{l}+1\right)\right] \cap A \neq \emptyset$ for all $l \in \mathbb{N}$. Now select natural numbers $\theta(l)$ and $\rho(l)$ such that $\beta(\theta(l)) \in\left(\gamma\left(n_{l}\right), \gamma\left(n_{l}+1\right)\right]$ and $\rho(l) \in A \cap\left(\gamma\left(n_{l}\right), \gamma\left(n_{l}+1\right)\right]$ for all $l \in N$. Note that, since $x \in G(\gamma), x_{\beta(\theta(l))}=x_{\rho(l)}$ for all $l$ and, since $\rho(l) \in A, \lim _{l} x_{\rho(l)}=0$. Hence every subsequence of $x$ has a subsequence which converges to 0 and thus $x$ converges to 0 .

The equivalence of (2) and (3) follows immediately from noting that, if $B=$ $\bigcup_{r \in \mathbb{N}}\left(\gamma\left(n_{r}\right), \gamma\left(n_{r}+1\right)\right]$, then $T-\lim I_{B}=0$ if and only if $\limsup \sum_{r} \sum_{k>\gamma\left(n_{r}\right)}^{\gamma\left(n_{r}+1\right)} t_{n, k}=0$.

\section{AN APPLICATION TO WEIGHTED MEAN SUMMABILITY}

In this section we apply the results of the preceding sections to weighted mean summability methods generated by positive sequences. Let $\left\langle p_{k}\right\rangle$ be a sequence of nonnegative real numbers with $p_{1}>0$ and set $P_{n}=\sum_{k=1}^{n} p_{k}$. The weighted mean summability matrix $(R, p)$ is defined by $(R, p)_{n, k}=p_{k} / P_{n}$ for $k \leqslant n$ and $(R, p)_{n, k}=0$ otherwise.

Suppose that $p_{k}>0$ for all $k$. Then $(R, p)$ is a triangle and hence its convergence domain is can be regarded as a BK space where, if $x$ is in the convergence domain of $(R, p),\|x\|=\sup _{n} P_{n}^{-1}\left|\sum_{k=1}^{n} x_{k} p_{k}\right|$. This is a monotone norm: Since $\left\langle P_{n}\right\rangle$ is an increasing sequence, there is an $l \leqslant n$ such that $\left\|\sum_{k=1}^{n} x_{k} e^{k}\right\|=P_{l}^{-1}\left|\sum_{k=1}^{l} x_{k} p_{k}\right|$. It now follows from the definition of the norm that

$$
\left\|\sum_{k=1}^{n+1} x_{k} e^{k}\right\| \geqslant P_{l}^{-1}\left|\sum_{k=1}^{l} x_{k} p_{k}\right|=\left\|\sum_{k=1}^{n} x_{k} e^{k}\right\|
$$

and that $\|x\|=\sup _{n}\left\|\sum_{1}^{n} x_{k} e^{k}\right\|$. It is also straightforward to verify that $(R, p)$ is a type-M summability method and hence, if $(R, p)$ is regular, its convergence domain is a perfect coregular $B K$ space $[13$, p.42].

THEOREM 3.1. Let $p=\left\langle p_{k}\right\rangle$ be a sequence of positive numbers and suppose that $(R, p)$ is a regular weighted mean summability matrix. The following statements are equivalent.

(1) $G(\gamma)$ is a gap Tauberian condition for $(R, p)$ summability

(2) $G(\gamma)$ is a gap Tauberian condition for strong $(R, p)$ summability

(3) $\inf _{r} P_{\gamma(r)}^{-1} \sum_{k>\gamma(r-1)}^{\gamma(r)} p_{k}>0$. 
Proof: (1) implies (2) follows immediately from the observation that, for any nonnegative regular summability method $T$, if a sequence $x$ is strongly $T$-summable to $\lambda$ then $T-\lim x=\lambda$.

(2) implies (3): Suppose that $\inf _{r} P_{\gamma(r)}^{-1} \sum_{k>\gamma(r-1)}^{\gamma(r)} p_{k}=0$. Let $\left\langle\delta_{n}\right\rangle$ be a sequence of postive numbers decreasing monotonically to 0 . Select $r_{1}$ such that $P_{\gamma\left(r_{1}\right)}^{-1} \sum_{k>\gamma\left(r_{1}-1\right)}^{\gamma\left(r_{1}\right)} p_{k}<$ $\delta_{1}$. Now suppose that $n>1$ and $r_{1}<\cdots<r_{n-1}$ have been selected. First, using the hypothesis that $(R, p)$ is regular, select $N$ such that $l>N$ implies that

$$
P_{l}^{-1} \sum_{i=1}^{n-1} \sum_{k>\gamma\left(r_{i}-1\right)}^{\gamma\left(r_{i}\right)} p_{k}<\delta_{n} / 2
$$

Now select $r_{n}$ such that $\gamma\left(r_{n}-1\right)>N$ and $P_{\gamma\left(r_{n}\right)}^{-1} \sum_{k>\gamma\left(r_{n}-1\right)}^{\gamma\left(r_{n}\right)} p_{k}<\delta_{n} / 2$. Note that

$$
P_{\gamma\left(r_{n}\right)}^{-1} \sum_{i=1}^{n} \sum_{k>\gamma\left(r_{i}-1\right)}^{\gamma\left(r_{i}\right)} p_{k}<\delta_{n}
$$

An elementary computation using the definition of $(R, p)$ yields that if $l \in\left[\gamma\left(r_{n}-1\right), \gamma\left(r_{n+1}\right)\right)$, then

$$
\sum_{i=1}^{\infty} \sum_{k>\gamma\left(r_{i}\right)}^{\gamma\left(r_{i}+1\right)}(R, p)_{l, k} \leqslant P_{\gamma\left(r_{n}\right)}^{-1} \sum_{i=1}^{n} \sum_{k>\gamma\left(r_{i}-1\right)}^{\gamma\left(r_{i}\right)} p_{k}<\delta_{n} .
$$

It follows that

$$
\underset{n}{\limsup } \sum_{i=1}^{\infty} \sum_{k>\gamma\left(r_{i}\right)}^{\gamma\left(r_{i}+1\right)}(R, p)_{n, k}=0
$$

and hence, by $2.2(3), G(\gamma)$ is not a gap Tauberian condition for strong $(R, p)$ summability.

(3) implies (1): Note that

$$
\inf _{r}\left\|b^{r-1}(\gamma)\right\| \geqslant \inf _{r} P_{\gamma(r)}^{-1} \sum_{k>\gamma(r-1)}^{\gamma(r)} p_{k}>0
$$

and, since $(R, p)$ is regular, it follows that $\left\langle b^{r}(\gamma)\right\rangle$ is equivalent to $c_{0}$ 's unit vector basis. The result now follows from 1.2 . 
Corollary 3.2. $G(\gamma)$ is a gap Tauberian condition for $(R, p)$ if there is a $\delta>0$ such that

$$
\frac{\sum_{k>\gamma(r-1)}^{\gamma(r)} p_{k}}{\sum_{k=1}^{\gamma(r-1)} p_{k}}>\delta
$$

for all $r \in \mathbf{N}$.

REMARKs. 1. Recall that a conservative BK space $E$ is conull if it is not coregular, that is, $f(e)=\sum_{k} f\left(e^{k}\right)$ for all $f \in E^{\prime}$. It has been shown in [3] that, for any $\gamma$ and any conull BK space $E, G(\gamma)$ is never a gap Tauberian condition for $E$. It turns out, however, that one can establish an analog of a gap Tauberian theorem for a large class of conservative conull BK spaces.

Let $r=\left\langle r_{n}\right\rangle$ be an increasing sequence of natural numbers with $r_{1}=1$. For $x \in \omega$, let

$$
O_{n}^{r}(x)=\max \left\{\left|x_{u}-x_{v}\right|: r_{n} \leqslant u<v \leqslant r_{n+1}\right\}
$$

and set

$$
\Omega(r)=\left\{x \in \omega: \lim _{n} O_{n}^{r}(x)=0\right\} .
$$

If we define $\|x\|_{r}=x_{1}+\sup _{n} O_{n}^{r}(x)$, then $\left(\Omega(r),\|\|_{r}\right)$ is a conservative conull BK space $[13$, p.95]. Also, given an arbitrary conservative conull BK space $E$, there is an $r$ such that $\Omega(r) \subset E$.

Now define $Z(r) \subset \omega$ by

$$
Z(r)=\left\{x: \text { there is a scalar } \lambda \text { such that } x_{k}=\lambda \text { if } r_{2 n}<j \leqslant r_{2 n+1} \text { for all } n \in \mathrm{N}\right\} \text {. }
$$

An elementary $\varepsilon-N$ argument yields that $Z(r) \cap \Omega(r) \subset c$, which we record via the following proposition.

Proposition 4.1. $Z(r)$ is a Tauberian condition for $\Omega(r)$.

This is analogous to a gap Tauberian theorem in that $\left\langle\sum_{j>r_{2 n}}^{r_{2 n+1}} e^{j}: n \in \mathrm{N}\right\rangle$ is equivalent to $c_{0}$ 's unit vector basis in $\Omega(r)$.

2. Let $T$ be a nonnegative regular summability matrix and $x=\left\langle x_{k}\right\rangle \in \omega$. For each $\varepsilon>0$ and $\lambda \in \mathbf{R}$, let $A(\varepsilon, \lambda)=\left\{k:\left|x_{k}-\lambda\right| \geqslant \varepsilon\right\}$. The sequence $x$ is said to be $T$-statistically convergent to $\lambda$ if $T-\lim I_{A(e, \lambda)}=0$ for all $\varepsilon>0$. It is known that if $x$ is strongly $T$-summable to $\lambda$ then $x$ is $T$-statistically convergent to $\lambda$ and, if $x$ is bounded and $T$-statistically convergent to $\lambda$, then $x$ is strongly $T$-summable to $\lambda[2]$. 
The above observations allow us to conclude that $G(\gamma)$ is a gap Tauberian condition for $T$-statistical convergence if and only if $G(\gamma)$ is a gap Tauberian condition for strong $T$-summability. Since strong $T$-summability implies $T$-statistical convergence, a gap Tauberian condition for $T$-statistical convergence must also be a gap Tauberian condition for strong $T$-summability. To establish the converse, suppose $G(\gamma)$ is a gap Tauberian condition for strong $T$-summability and that $x \in G(\gamma)$ is $T$-statistically convergent to $\lambda$. Define $\tilde{x}$ by $\widetilde{x}_{k}=x_{k}$ if $\left|x_{k}-\lambda\right|<1$ and $\tilde{x}_{k}=\lambda+1$ otherwise. Note that $\vec{x}$ is bounded and statistically convergent to $\lambda$ and hence strongly $T$-summable to $\lambda$. Now, since $\tilde{x} \in G(\gamma), \tilde{x}$, and hence $x$, is convergent to $\lambda$.

When we restrict our attention to $(R, p)$ where $p_{k}=1$ for all $k,(R, p)$ is the Cesaro mean (order 1 ) and $(R, p)$-statistical convergence is the usual statistical convergence as discussed in [7] and [11]. Theorem 3.1 yields Fridy's result that $G(\gamma)$ is a gap Tauberian condition for statistical convergence if $\liminf _{n} \gamma(n+1) / \gamma(n)>1$ and its converse.

3. If we recall a few elementary facts about the Stone-Čech compactification of $\mathbf{N}$, the result of section 2 can be given an interpretation in the setting of $\mathbf{N}^{*}=\beta \mathbf{N}-\mathbf{N}$. Recall that $\beta \mathrm{N}$ can be identified with the set of all ultrafilters on $N[12,8]$. If $p$ is an ultrafilter on $\mathbf{N}$, we say that $p$ is a free ultrafilter if $p$ does not contain a bounded subset of $\mathbf{N}$ and recall that $\mathbf{N}^{*}$ can be identified with the collection of all free ultrafilters on $\mathbf{N}$. If $A \subset \mathbf{N}$, we let $A^{*}=\left\{p \in \mathbf{N}^{*} \mid A \in p\right\}$ and recall that $\left\{A^{*} \mid A \subset \mathbf{N}\right\}$ is a base of clopen sets for the relative topology on $N^{*}$. Also recall that if $x=\left\langle x_{n}\right\rangle \in l_{\infty}$, then $x$ can be extended to $x^{\beta} \in C(\beta N)$ by defining $x^{\beta}(p)=\cap\left\{c l_{\mathbf{R}}\left(x^{\rightarrow}(A)\right) \mid A \in p\right\}$ for an ultrafilter $p$ on $N$ and that, for a sequence $x \in l_{\infty}, \lim _{k} x_{k}=\lambda$ if and only if $x^{\beta} \mid \mathbf{N}^{*}=\lambda$.

If $T$ is a nonnegative regular summability matrix, the support set $K_{T}$ of $T$ is defined by

$$
K_{T}=\bigcap\left\{A^{*} \mid A \subset \mathrm{N}, \lim _{n} \sum_{k} t_{n, k} I_{A}(k)=1\right\}
$$

The support set of a matrix was introduced in [9] and has been discussed by a number of authors. Note that $p \in K_{T}$ if and only if $p \in A^{*}$ whenever $T-\lim I_{A}=1$. As was noted in [1], Theorem 2.1 can be reformulated to yield that $K_{T}$ is a $P$-set in $\mathrm{N}^{*}$ (that is, $K_{T}$ is interior to any $G_{\delta}$ that contains $K_{T}$ ). It can also be used to show that if $x \in l_{\infty}$, then $x$ is strongly $T$-summable to $\lambda$ if and only if $x^{\beta} \mid K_{T}=\lambda$. It follows that $G(\gamma)$ is a gap Tauberian condition for bounded strong $T$-summability if and only if $x \in G(\gamma) \cap l_{\infty}$ and $x^{\beta} \mid K_{T}=\lambda$ implies that $x^{\beta} \mid \mathrm{N}^{*}=\lambda$.

ThEOREM 4.2. $G(\gamma)$ is a gap Tauberian condition for strong $T$-summability if 
and only if

$$
c l_{N *}\left(\bigcup_{r \in N}\left(\gamma\left(n_{r}\right), \gamma\left(n_{r}+1\right)\right]\right) \cap K_{T} \neq 0
$$

for any increasing sequence of integers $\left\langle n_{r}\right\rangle$.

PROOF: Observe that $c l_{\mathbf{N}^{*}}\left(\bigcup_{k \in \mathbb{N}}\left(\gamma\left(n_{r}\right), \gamma\left(n_{r}+1\right)\right]\right) \cap A^{*} \neq \emptyset$ for all $A \subset \mathbf{N}$ such that $T-\lim I_{A}=1$ if and only if $\limsup \sum_{r} \sum_{r>\gamma\left(n_{r}\right)}^{\gamma\left(n_{r}+1\right)} t_{n, k}>0$. The result now follows from $2.2(3)$.

\section{REFERENCES}

[1] R. Atalla, 'On the multiplicative behaviour of regular matrices', Proc. Amer. Math. Soc. 26 (1970), 437-446.

[2] J. Connor, 'Two valued measures and summability', Analysis 10 (1990), 373-385.

[3] J. Connor and A.K. Snyder, 'Tauberian theorems for conull spaces', Intermat. J. Math. Math. Sci. 8 (1985), 689-692.

[4] J. Diestel, Sequences and series in Banach spaces (Springer-Verlag, Berlin, Heidelberg, New York, 1984).

[5] A.R. Freedman and J.J. Sember, 'Densities and summability', Pacific J. Math. 95 (1981), 293-305.

[6] J. Fridy, 'Tauberian theorems via block dominated matrices', Pacific J. Math. 81 (1979), 81-91.

[7] J. Fridy, 'On statistical convergence', Analysis 5 (1985), 301-313.

[8] L. Gillman and M. Jerison, Rings of continuous functions, University Series in Higher Math. (Van Nostrand, Princeton, 1960).

[9] M. Henriksen, 'Multiplicative summability methods and the Stone-Cech compactification', Math. Z. 71 (1959), 427-435.

[10] J.D. Hill and W.T. Sledd, 'Approximation in bounded summability fields', Canad. J. Math. 20 (1968), 410-415.

[11] I.J. Maddox, 'A Tauberian theorem for statistical convergence', Math. Proc. Cambridge. Philos. Soc. 106 (1989), 277-280.

[12] W. Rudin, 'Homogeneity problems in the theory of Cech compactifications', Duke Math. J. 23 (1956), 409-420.

[13] A. Wilansky, Summability through functional analysis (North-Holland, Amsterdam, 1984).

Department of Mathematics

Ohio University

Athens OH 45701

United States of America 